\title{
Desafio do egresso de enfermagem para inserção no mercado de trabalho
}

\author{
Challenge of nursing graduates to enter the labor market
}

Reto de los egresados de enfermería para ingresar al mercado laboral

\author{
Pamela Borges Mello $^{1 *}$, Lilía Marques Simões Rodrigues², Marilei de Melo Tavares ${ }^{3}$, Eliara Adelino \\ da Silva ${ }^{4}$, Thiago Augusto Silva ${ }^{5}$, Denise Duarte Celento ${ }^{6}$
}

Como citar esse artigo. Mello, PB; Rodrigues; LMS; Tavares, MM; da SIlva, EA; Silva, TA; Celento, DD. Desafio do egresso de enfermagem para inserção no mercado de trabalho. Revista Pró-UniverSUS. 2021 Jul./Dez.; 12 (2)SUPLEMENTO: 47 - 52

\section{Resumo}

No período de graduação os estudantes de enfermagem sonham e criam grandes expectativas para o momento em que possam ingressar no mercado de trabalho, conquistar um emprego e poder praticar todo conhecimento adquirido durante os anos, se torna o maior desejo do aluno assim que conclui a graduação, no entanto encontram grandes dificuldades no início da carreira. O estudo tem como objetivo indentificar as dificuldades dos egressos de enfermagem na sua inserção ao mercado de trabalho a partir da literatura existente. Trata-se de uma revisão de literatura, realizada em base de dados LILACS, Scientific Eletonic Library Online (SciELO), no período de 2011 a 2020, foi utilizado o operador Booleano AND junto com os seguintes descritores: enfermagem; egressos; mercado de trabalho. Os resultados apontam para expectativas dos egressos de enfermagem: dilemas e desafios; e para o processo formativo - ampliando oportunidades para o mercado trabalho em enfermagem. Percebese que existe uma grande dificuldade dos egressos de enfermagem na sua inserção no mercado de trabalho. Conclui-se que é necessário realizar pesquisas sobre este tema para assim conseguir indentificar as dificuldades do egresso na sua prática profissional e criar métodos que auxiliarão os recém-formados neste período de insersão no mercado de trabalho.

Palavras-chave: Enfermagem; Egressos; Mercado de Trabalho.

\begin{abstract}
In the undergraduate period, nursing students dream and create high expectations for the moment when they can enter the job market, win a job and be able to practice all the knowledge acquired over the years, it becomes the student's greatest desire as soon as they graduate, however, they encounter great difficulties at the beginning of their careers. The study aims to identify the difficulties of nursing graduates in their insertion into the job market from the existing literature. It is a literature review, carried out in LILACS database, Scientific Eletonic Library Online (SciELO), MEDLINE, in the period from 2011 to 2020, the Boolean operator was used AND together with the following descriptors: nursing; graduates; job market. The results point to expectations of nursing graduates: dilemmas and challenges; and for the training process - expanding opportunitteis for the nursing work market. It is noticed that there is a great difficulty for nursing graduates in their insertion in the job market. It is concluded that it is necessary to carry out research on this theme in order to be able to identify the difficulties of the graduate in his professional practice and create methods that helped the recent graduates in this period of insertion in the labor market.

Keywords: Nursing; Graduates; Job market.
\end{abstract}

Afiliação dos autores: ${ }^{1 *}$ Acadêmica da graduação em enfermagem pela Universidade de Vassouras, Vassouras, Rio de Janeiro, Brasil. ORCID https://orcid.org/0000-0002-6327-1823. ${ }^{2}$ Enfermeira. Mestre em Enfermagem pela UFF. Professora e Coordenadora do Curso de Graduação em Enfermagem da Universidade de Vassouras, RJ, Brasil. ORCID: https://orcid. org/0000-0003-2979-6316.

${ }^{3}$ Psicóloga. Pós-Doutora pela UERJ. Docente do Curso de Enfermagem da Universidade de Vassouras. Vassouras, RJ, Brasil. ORCID: http://orcid.org/0000-0002-3276-0026.

${ }^{4}$ Enfermeira e Terapeuta Holística. Mestre em Ciências da Saúde e Meio Ambiente pela UNIFOA. Professora do Curso de Enfermagem da Universidade de Vassouras, Vassouras, RJ, Brasil. ORCID: https://orcid.org/0000-0003-4387-6662.

${ }^{5}$ Enfermeiro. Doutor em Enfermagem pela Escola de Enfermagem Anna Nery-UFRJ. Docente do Curso de Enfermagem da Universidade de Vassouras, Vassouras, RJ, Brasil. OR-

CID: https://orcid.org/0000-0001-6870-5101.

${ }^{6}$ Administradora. Mestre em Ciências do Cuidado em Saúde pela UFF. Professora do Curso de Enfermagem da Universidade de Vassouras, Vassouras, RJ, Brasil. ORCID: https:// orcid.org/0000-0002-1829-759X.

* Email de correspondencia: pamborgmello@hotmail.com

Recebido em: 21/01/21. Aceito em: 09/06/21. 


\section{Resumen}

En la etapa de pregrado, los estudiantes de enfermería sueñan y crean altas expectativas para el momento en que puedan ingresar al mercado laboral, ganar un puesto de trabajo y poder practicar todos los conocimientos adquiridos a lo largo de los años, se convierte en el mayor anhelo del estudiante nada más graduarse. sin embargo, encuentran grandes dificultades al comienzo de sus carreras. El estudio tiene como objetivo identificar las dificultades de los egresados de enfermería en su inserción al mercado laboral a partir de la literatura existente. Se trata de una revisión de la literatura, realizada en la base de datos LILACS, Scientific Eletonic Library Online (SciELO), MEDLINE, en el período de 2011 a 2020, se utilizó el operador booleano AND junto con los siguientes descriptores: enfermería; graduados; mercado de trabajo. Los resultados apuntan a las expectativas de los egresados de enfermería: dilemas y desafíos; y para el proceso de formación - ampliando las oportunidades para el mercado laboral de enfermería. Se advierte que existe una gran dificultad para los egresados de enfermería en su inserción en el mercado laboral. Se concluye que es necesario realizar una investigación sobre este tema para poder identificar las dificultades del egresado en su práctica profesional y generar métodos que ayuden a los recién egresados en este período de inserción en el mercado laboral..

Palabras clave: Enfermería; Graduados; Mercado de trabajo.

\section{Introdução}

No decorrer do curso de graduação, estudantes de enfermagem sonham e criam grandes expectativas para o momento em que possam ingressar no mercado de trabalho, conquistar um emprego e poder praticar todo conhecimento adquirido durante os anos. Assim ingressar no mercado de trabalho torna-se o maior desejo do aluno assim que conclui a graduação.

O início da carreira apresenta obstáculos para o profissional de enfermagem, como a falta de experiência técnica para a realização de procedimentos, a dificuldade para liderar a sua equipe, a insegurança e o medo fazem parte desse momento.

É notório que os acadêmicos de enfermagem indealizem formas de como se sobressair frente a equipe de enfermagem e realizar-se profissionalmente. Idealizam cuidar das pessoas, resolver problemas, promover e garantir a saúde e o bem-estar dos seus pacientes, mas frequentemente a realidade se torna um pouco diferente do esperado, quando encontram a vivência da rotina das instituições, muitos sonhos planejados durante o seu processo de formação são capturados pelos serviços institucinalizados, e, com isso ficam desestimulados ${ }^{1}$

A enfermagem possui um papel de grande importancia para a saúde do Brasil, o enfermeiro não possui função apenas para a assistencia direta ao paciente, ele também possui hablidades para gerenciamento e adminstração hospitalar, na eduação em saúde, na assistencia doimiciliar, na saúde pública e também podemos observar nos ultimos anos um grande crescimento da enfermagem nas empresas e no empreendedorismo.

No que diz respeito à gerência, as empresas têm exigido profissionais que respondam rapidamente às atuais exigências do mercado cada vez mais competitivo2. É de reponsabilidade do profissional, as atualizações através de cursos, palestras, workshops e treinamentos para desenvolver habilidades de resoluções de conflitos, relacionamentos e liderança.

O enfermeiro deve ter competência para liderar e inspirar os seus liderados a exercerem o seu trabalho de modo ético/profissional, para que isso ocorra é necssário a criação de laços de confiança, e compreenssão da teoria das relações humanas que auxiliará ao enfermeiro como coordenar a sua equipe ${ }^{3}$. Em muitos cenários o enfermeiro possui função assistencial e gerencial, possuindo uma melhor liderança com sua equipe irá fazer com que tenha maiores e melhores resultados, proporciando assim uma melhor qualidade de vida para o paciente e a satisfação da equipe de enfermagem.

$\mathrm{O}$ ensino de Enfermagem, provavelmente, por historicamente ter-se atrelado ao ensino médico, guiouse por um modelo que tem o hospital como cenário prioritário de práticas e formação, mas é necessário compreender que a formação do acadêmico deve ser ampla, auxiliando assim ao desenvolvimento de outras habilidades ${ }^{3}$.

O enfermeiro também pode se inserir dentro do Programa de Saúde da Família (PSF), que foi criada em 1994 pelo Ministério da Saúde, neste ambiente o enfermeiro possui atributos tanto na assistência ao paciente, como também gestão da equipe. O que demonstra que além de cuidar da saúde daquela comunidade, ele também precisa supervisionar toda a equipe e adminstrar a unidade de saúde ${ }^{4}$.

O enfermeiro que atua na atenção básica possui tanto a responsabilidade na assistência como também na gerencia do local, o que faz com que necessite desenvolver habilidades para atuar nas duas áreas. Por vezes, na graduação o estudante tem preparo para assumir tais responsabilidades é aplicado à realização de técnicas, mas não à resolução de conflitos que possam surgir no ambiente de trabalho. A formação não deve priveligiar apenas o ingresso do enfermeiro no mercado de trabalho mas também sua adaptação nele. Assim torna-se necessário que durante sua formação seja enfatizado o desenvolvimento de competências para a liderança, gestão de pessoas e relações interpessoais ${ }^{5}$. 
A formação do enfermeiro tem sido debatida no cenário nacional e muitos problemas já foram identificados, sendo abordado a necessidade de revisar e atualizar as disciplinas e conteúdos do currículo da graduação ${ }^{6}$, pois é importante que elas estejam dentro da realidade do nosso país.

Conhecer as dificuldades vivenciadas por egressos de enfermagem no mercado de trabalho é uma forma de analisar, compreender e refletir sobre questões relativas ao ensino superior, assim como os aspectos inerentes ao mercado de trabalho ${ }^{5}$. Por conta dessa observação, a importância de pesquisar sobre a temática, trás como objetivo indentificar as dificuldades dos egressos de enfermagem na sua inserção ao mercado de trabalho a partir da literatura existente.

\section{Metodologia}

Trata-se de uma revisão da literatura, que inclui a análise de pesquisas relevantes que dão suporte para a tomada de decisão, permitindo a incorporação desses achados na prática clínica. Este tipo de estudo é uma estratégia para a identificação e análise das evidências existentes de práticas de saúde, quando a produção de conhecimento científico não está suficientemente fundamentada. ${ }^{7-8}$

O levantamento bibliográfico foi realizado em ambiente virtual na Biblioteca Virtual de Saúde (BVS), na base: Lilacs e Scielo, durante os meses de setembro a dezembro de 2020. As obras foram pesquisadas por meio dos seguintes descritores: enfermagem and egressos and mercado de trabalho.

Foram selecionados para este estudo artigos que, na leitura guardavam relação direta com o tema em questão. Os critérios de inclusão propostos foram: periódicos da área de enfermagem publicados no Brasil entre os anos de 2011 a 2020, em língua portuguesa, em forma de texto completo e que estavam disponíveis nos locais selecionados para a coleta.

Visto que o objeto do estudo reside nas dificuldades dos egressos de enfermagem para inserção no trabalho. No levantamento inicial na BVS foram obtidos 65 artigos. Após leitura inicial dos titulos e resumos foram selecionados 18, e aplicados os critérios de inclusão propostos no estudo, sendo selecionados 7 artigos que serviram para este estudo.

Em seguida foi feita a relação entre o conteúdo, título, resumo, e se atendiam ao objeto do presente estudo - com atenção especial para os resultados e conclusão das mesmas, pois são nestes quesitos que encontramos a real propriedade do estudo realizado.

Após a seleção dos artigos partimos para análise, com recortes temáticos de conteúdo, visando identificar os temas encontrados em nossa pesquisa bibliográfica, identificando, desse modo, algumas recorrências. Posteriormente, foram agrupados e reagrupados alguns recortes temáticos similares.

Em seguida organizou-se um grupo temático, principais dificuldades apresentadas por egressos de enfermagem para inserção no mercado de trabalho, atentando para o que de mais relevante foi identificado nos estudos. Baseando-se nesse contexto, elaboramos e nomeamos as seguintes categorias: $\left(1^{\mathrm{a}}\right)$ Expectativas dos egressos de enfermagem: Dilemas e Desafios; $\left(2^{\mathrm{a}}\right)$ Processo formativo - ampliando a oportunidade para o mercado trabalho em enfermagem.

\section{Resultados e Discussão}

Foram analisados no presente estudo 7 artigos científicos publicados no ambiente virtual na Biblioteca Virtual de Saúde (BVS), na base: Lilacs e Scielo entre os anos de 2011 a 2020, que abordavam os descritores enfermagem, egressos e mercado de trabalho. Em seguida após a leitura de todos os artigos, fizemos uma seleção do material quanto ao ano de publicação e o tipo de metodologia aplicada. Após a caracterização deste material organizou-se duas categorias: Expectativas dos egressos de enfermagem: Dilemas e Desafios; Processo formativo, ampliando oportunidades para o mercado de trabalho em enfermagem.

Dos 7 artigos selecionados observou-se nessa revisão integrativa que um (14,28\%) dos artigos selecionados do ano de 2012; dois $(28,57 \%)$ do ano de 2014; dois $(28,57 \%)$ do ano de 2017 ; dois $(28,57 \%)$ do ano de 2019. Percebe-se que os dados da pesquisa estão bem recentes, visto que os maiores números de materiais são do ano de 2017 e 2019, percebe-se ainda que houve um período de 3 anos, de 2014 a 2017 sem a realização de estudos sobre o tema, o que demonstra a existência de poucas pesquisas acerca do tema o que reforça a necessidade de maiores explorações para auxílio aos egressos, diante as dificuldades que possam ser encontradas na sua inserção ao mercado de trabalho.

Com relação a metodologia aplicada nos artigos, observou-se que seis dos artigos selecionados são de naturaza descritiva e um discurso do sujeito coletivo. Sendo que todos os 7 artigos selecionados obtiveram os resultados de suas pesquisas por meio de coleta de dados, correspondendo sete por meio de questionários eletrônicos e três por meio de entrevistas. Isso demostra que a presente pesquisa obteve análise de relatos e experiências dos profissionais de enfermagem na sua inserção ao mercado de trabalho.

Para melhor ilustrar os resultados o quadro 1 abaixo: 
Quadro 1. Distribuição dos artigos selecionados para pesquisa.

\begin{tabular}{|c|c|c|c|c|}
\hline Produção Científica & Ano & Autor & Tipo de Estudo & Revista \\
\hline $\begin{array}{l}\text { O enfermeiro no mercado de } \\
\text { trabalho: inserção,competências e } \\
\text { habilidades. }\end{array}$ & 2017 & $\begin{array}{l}\text { Püschel,VAA; } \\
\text { Costa,D; Reis PP; } \\
\text { Oliveira LB; } \\
\text { Carbogim, FC }\end{array}$ & $\begin{array}{l}\text { Estudo } \\
\text { Exploratório } \\
\text { Descritivo }\end{array}$ & $\begin{array}{l}\text { Revista Brasileira de } \\
\text { Enfermagem,v.70, n.6, } \\
\text { p. 1220-1226, 2017. }\end{array}$ \\
\hline $\begin{array}{l}\text { Perfil de egressos de Enfermagem: } \\
\text { competências e inserção } \\
\text { profissional }\end{array}$ & 2019 & $\begin{array}{l}\text { Barbosa ASC; Luiz } \\
\text { FC; } \\
\text { Friedrich DBC; } \\
\text { Farah, BF; } \\
\text { Carbogim, FC }\end{array}$ & $\begin{array}{l}\text { Estudo } \\
\text { Exploratório } \\
\text { Descritivo }\end{array}$ & $\begin{array}{lr}\text { Revista } & \text { Latino- } \\
\text { Americana } & \text { de } \\
\text { Enfermagem, v. } 27, \\
2019 .\end{array}$ \\
\hline $\begin{array}{lll}\text { Percepções de egressos } & \text { de } \\
\text { enfermagem frente a inserção no } & \\
\text { mercado de trabalho } & & \end{array}$ & 2014 & $\begin{array}{l}\text { Cambiriba,TFC; } \\
\text { Ferronato, } \mathrm{AF} ; \\
\text { Fontes, } \mathrm{KB}^{11}\end{array}$ & $\begin{array}{l}\text { Estudo } \\
\text { Exploratório } \\
\text { Descritivo }\end{array}$ & $\begin{array}{l}\text { Arquivos de Ciências } \\
\text { da Saúde da UNIPAR, } \\
\text { v. } 18 \text {, n. } 1,2014 .\end{array}$ \\
\hline $\begin{array}{l}\text { Formação profissional e inserção } \\
\text { no mercado de trabalho: } \\
\text { percepções de egressos de } \\
\text { graduação em enfermagem. }\end{array}$ & 2012 & $\begin{array}{l}\text { Colenci, R; } \\
\text { Berti, } \mathrm{HW}^{12}\end{array}$ & $\begin{array}{l}\text { Discurso do } \\
\text { sujeito coletivo }\end{array}$ & $\begin{array}{l}\text { Revista da Escola de } \\
\text { Enfermagem da USP, } \\
\text { v. } 46 \text {, n. 1, p. 158-166, } \\
2012 \text {. }\end{array}$ \\
\hline $\begin{array}{l}\text { Processo de formação e inserção } \\
\text { no mercado de trabalho: uma visão } \\
\text { dos egressos de enfermagem. }\end{array}$ & 2014 & $\begin{array}{l}\text { Canever, BP; } \\
\text { Gomes, DC; } \\
\text { Jesus, BH; } \\
\text { Spillere, LB; } \\
\text { Prado, ML; } \\
\text { Backes, VMS }\end{array}$ & $\begin{array}{l}\text { Estudo } \\
\text { Exploratório } \\
\text { Descritivo }\end{array}$ & $\begin{array}{l}\text { Revista Gaúcha de } \\
\text { Enfermagem, v. } 35 \text {, n. } \\
\text { 1, p. 87-93, } 2014\end{array}$ \\
\hline $\begin{array}{l}\text { Estágio extracurricular de } \\
\text { Enfermagem: estratégia para a } \\
\text { formação profissional }\end{array}$ & 2019 & $\begin{array}{l}\text { Silva, ANC; } \\
\text { Moreira, DP; } \\
\text { Freitas, CMA } \\
\text { Teixeira, AKS; } \\
\text { Pinheiro, ARM }{ }^{14}\end{array}$ & $\begin{array}{l}\text { Estudo } \\
\text { Exploratório } \\
\text { Descritivo }\end{array}$ & $\begin{array}{lr}\text { Enferm. } & \text { foco } \\
\text { (Brasília), p. } & 129-135, \\
2019 . & \end{array}$ \\
\hline $\begin{array}{l}\text { Enfermeiros egressos do currículo } \\
\text { integrado: inserção e atuação } \\
\text { profissional. }\end{array}$ & 2017 & $\begin{array}{l}\text { Costa,TV; } \\
\text { Guariente, } \\
\text { MHDM. }^{15}\end{array}$ & $\begin{array}{l}\text { Estudo } \\
\text { Exploratório } \\
\text { Descritivo }\end{array}$ & $\begin{array}{l}\text { Rev. enferm. UFPE on } \\
\text { line, p. } 77-85,2017 .\end{array}$ \\
\hline
\end{tabular}

\section{Expectativas dos egressos de enfermagem para o trabalho: Dilemas e Desafios}

O desejo de exercer todo o conhecimento adquirido durante a graduação gera no egresso uma grande expectativa para a sua inserção ao mercado de trabalho. Porém os recém-formados se deparam com muitas dificuldades neste momento.

De acordo com a literatura selecionada e analisada as maiores dificuldades encotradas pelos egressos de enfermagem na sua inserção ao mercado de trabalho foram: falta de experiência $9,10,11,12,13$; falta de especialização ${ }^{9,10}$; remuneração ${ }^{10,12}$; saturação do mercado de trabalho ${ }^{10,11}$ e concorrência ${ }^{12}$. Apesar de todas as dificuldades encontradas percebe-se que os egressos conseguem entrar ao mercado de trabalho rapidamente, os estudos ${ }^{14,10}$ apontam que mais de $50 \%$ dos entrevistados se inseriram na profissão entre seis meses e em menos de doze meses ${ }^{10,15}$ após a formação, demosntrando que apesar de todas as dificuldades, existem oportunidades para os recém-formados.

O mercado de trabalho na área da enfermagem é amplo, o enfermeiro pode exercer sua profissão em diversos cenários, sendo eles em ambiente Hospitalar, nas Unidades Básicas de Saúde (UBS), nos atendimentos domiciliares, nos ensinos superiores, em empresas, de forma autônoma e diversas outras especializações existentes. Essa amplitude auxilia o profissional a entrar no mercado de trabalho, correspondendo que a maioria dos profissionais trabalham nos Hospitais ${ }^{9,12}$ e nas $\mathrm{UBS}^{11}$, sendo que nas Unidades Hospitalar requerem mais enfermeiros do que nas unidades básicas, com isso o hospital será o principal cenário para contratação de profissionais.

Segundo estudo realizado em Minas Gerais, 56\% dos entrevistados que possuiam emprego na área atuava na assistência de enfermagem ${ }^{10}$, o que demostra a grande necessidade dos egressos de enfermagem possuirem habilidades para prestar assistência, pois a grande maioria de vagas de empregos são nessa categoria, por conta disso é importante adquirir habilidades na tentativa de minimizar as dificuldades possivelmente encontradas.

A literatura aponta que alguns entrevistados relataram possuir dificuldades no primeiro emprego, 
descreveram não sentir segurança, distanciamento entre a prática ensinada e a prática profissional, dificuldades pela falta de conhecimentos específicos e insegurança para assumir uma equipe ${ }^{15}$. Esses desafios são presentes no cotidiano dos egressos frente ao primeiro emprego, relatando que os desafios não são apenas para inserção ao mercado de trabalho, mas também em saber lidar com conflitos presentes frente ao primeiro emprego.

Sendo que alguns desses desafios podem ser minimizados pela educação continuada com a realização de especializações, palestras, cursos e conferências, pois elas irão auxiliar os egressos de enfermagem a lidarem com suas dificuldades no seu primeiro contato com o mercado de trabalho.

\section{Processo formativo - ampliando oportunidades para o mercado trabalho em enfermagem}

Os requisitos do mercado de trabalho para a inserção de novos profissionais é crescente, fazendo com que os egressos aspirem, essencialmente por cursos de especialização e residências que possuem como objetivo a qualificação e aperfeiçoamento das habilidades técnicas9. Considerando que as dificuldades em relação a formação para a inserção no mercado de trabalho, como exigências de pós-graduação e/ou especialização na área, observando-se que ao terminar o curso é de grande relavância a continuação dos estudos pois auxiliarão na entrada ao mercado de trabalho, ampliando assim as oportunidades.

A DCN/ENF (Resolução CNE/CES nº 3 de 7 de novembro de 2001), estabeleceu como obrigatoriedade no curso de Enfermagem a realização de Estágio Supervisionado nos dois últimos períodos da graduação, abordando várias áreas de assistência da enfermagem, sendo em hospitais gerais, ambulatórios, UBS. Consistindo que a carga horária mínima do estágio curricular supervisionado deverá totalizar $20 \%$ (vinte por cento) da carga horária total do curso ${ }^{16}$.

Foi observado dentre os artigos analisados ${ }^{14}$, que o estágio extracurricular contribuiu de maneira positiva para a sua inserção ao mercado de trabalho, certificando assim que o estágio possibitou aos estudantes a formação de vínculos e a oportunidade de demonstrar suas habilidades, bem como seus conhecimentos, apresentando um bom desempenho, intensificando também suas experências e com isso aumentando as suas oportunidades para um novo emprego.

De acordo com a literatura ${ }^{13,11}$, disciplinas curriculares e estágio extracurricular destacam-se como oportunidade para o mercado de trabalho. Conteúdos e carga horária das disciplinas curriculares, realizadas na formação acadêmica foram relatados pelos egressos, como experiências significativa que contribuíram de modo marcante para à atuação profissional. $\mathrm{O}$ estágio extracurricular proporciona ao acadêmico experiências únicas que contribuiu para a sua formação ${ }^{13}$. A participação em estágio extracurricular também foi assinalada como fator colaborador para a conquista de um emprego ${ }^{10}$.

Contudo, em um estudo ${ }^{12}$ a maioria dos entrevistados criticaram o estágio curricular declarando que o campo era limitado a determinados hospitais e que não foi possível ver tudo que foi visto na teoria, como procedimentos mais complexos, demonstrando assim que muitas experiências acabam não sendo adquiridas no momento do estágio pois existem procedimentos que são realizados com menos frequência, e com isso existem expriências que podem ser adquiridas através de uma educação continuada podendo ser através de especializações e/ou através de estágios extracurriculares.

Ao passo que no estudo ${ }^{11}$, destaca-se que o estágio extracurricular proporciona maiores experiências ao egresso, diferentemente do estágio curricular que é realizado durante a graduação, pois nele o acadêmico possui menos oportunidades para se relacionar com as equipes e possui todo um planejamento e cronograma estimulado pela instituição. Demonstrando assim que o estágio extracurricular é apondado pelo egresso como um momento de maior aprendizado.

Em relação a pós graduação, os enfermeiros entrevistados em um estudo ${ }^{10}$, grande parte $(44,5 \%)$ estavam cursando ou já haviam cursado pós-graduação Stricto sensu, que referem-se aos programas de mestrado e doutorado e $43 \%$ estavam cursando ou já apresentavam especializações Lato sensu. Comprovando que a continuidade dos estudos mesmo após o final da graduação auxilia em uma melhor adesão ao mercado de trabalho. Compreendendo também a necessidade de realização de atualizações por meio de cursos e capacitações que auxiliam na valorização do curriculo lattes do profissional, aumentando as oportunidades no mercado de trabalho.

As atividades de extensão, monitoria e de pesquisa também auxiliam na construção de um profissional ético, crítico, com características de liderança e autonomia. Em um estudo sobre competências e inserção profissional ${ }^{10}$, os egressos de enfermagem relataram que atividades desenvolvidas durante a graduação relacionadas à extensão, monitaria, e a pesquisa, destacando a extensão universitária, colaboraram para uma formação crítica, criativa e com resolução de problemas, com foco para o atendimento em todos os âmbitos de saúde. Para tanto, é necessário uma maior inserção dos acadêmicos de enfermagem nessas atividades. O estudo ${ }^{10}$ também aponta que a graduação de enfermagem instrui o egresso a desempenhar algumas caracteristícas como a tomada de decisão e intervenção nos problemas de saúde a partir 
de uma assistência holística e satisfatória em todos os níveis de atenção a comunidade, contribuindo assim com a formação do enfermeiro.

\section{Conclusão}

Pretendia-se, com este estudo, uma apreciação das situações enfrentadas pelos egressos de Enfermagem quando da sua inserção no mercado de trabalho, buscando identificar as dificultades intrínsecas do processo. Considerando a metodologia aplicada, essas dificuldades foram identificadas.

Vale notar que exercer a profissão é um dos maiores desejos dos egressos de enfermagem ao saírem da graduação, mas na maioria das vezes eles encontram algumas dificuldades nesse primeiro contato com o mercado de trabalho. Dentre os fatores identificados, a falta de habilidades e especializações são alguns dos desafios encontrados ao sair da graduação, ao que contribui a insegurança dos recém-formados, bem como a falta de experiência no ambiente profissional e de tato para questões conflituosas e de gestão. Contudo, cabe a esses novos profissionais encontrar medidas para enfrentar estes dilemas, que serão resolvidos justamente no processo de aquisição da experiência profissional.

Por meio desta pesquisa foi observado que é de grande importância os egressos de enfermagem se inserirem em programas como residencia, pósgraduações e outros métodos de educação continuada, para adquirir conhecimentos, experiencias e agregar ao seu curriculo, trazendo assim um diferencial que irá auxiliar na sua inserção ao mercado de trabalho e nas resoluções das dificuldades que poderão ser encontradas

Por fim, é de fundamental importância realizar o aprofundamento de pesquisas sobre essa temática tão relevante mas pouco analisada. E assim auxiliar na indentificação das dificuldades do egresso na sua prática profissional, trazendo cada vez mais para o seu período de graduação discussões sobre sua formação, e o complemento da literatura para a criação de métodos que possam auxiliar os recém-formados neste momento de insersão no mercado de trabalho.

\section{Referências}

1. B1. Souza FA, Paiano M. Desafios e dificuldades enfrentadas pelos profissionais de enfermagem em início de carreira. Rev. Min. Enferm.2011Abr./Jun,; 15(2): 267-73.

2. Oliveira WA. Enfermagem: Os desafios e dificuldades do início de carreira. Rev. Refaci. 2017 Jan/Jul,; 2 (2).

3. Oliveira IR, Rodrigues LMS. Tipos de liderança adotados pelo enfermeiro no âmbito hospitalar. Revista Pró-UniverSUS. 2017 Jun./Dez.; 08 (2): $15-20$.

4. Rodrigues LMS. O planejamento do estágio supervisionado de enfermagem na atenção básica: avaliação da ação dialógiva ensino-serviço.
Niterói: Universidade Federal Fluminense, 2012. Dissertação Mestrado Profissional Enferagem Assistencial.

5. Cambiriba TFC, Ferronato AF, Fontes KB. Percepções de egressos de enfermagem frente a inserção no mercado de trabalho. Arq. Ciênc. Saúde Unipar. 2014 Jan/Abr,; 18 (1): 27-32.

6. Rodrigues LMS, Tavares CMM, Elias ADS. Interação, ensino e serviço de saúde para o desenvolvimento do estágio supervisionado em enfermagem na atenção básica. Revista de Pesquisa Cuidado é Fundamental. online. $2014 \mathrm{Jan} / \mathrm{marc}$; 6(1): 357-363.

7. Benefiel LE. Implementing evidence-based practine in home care. Home Healthc Nurce. 2003;21(12): 804-9.

8. Ribeiro RP, Martins JT, Marziale MHP, Robazzi MLCC. O adoecer pelo trabalho na enfermagem: uma revisão integrativa. Rev.esc.enferm. USP. 2012 Abr: 46(2): 495-504

9. Püschel VAA, Costa D, Reis PP, Oliveira LB, Carbogim FC. O enfermeiro no mercado de trabalho: inserção, competências e habilidades. Revista Brasileira de Enfermagem, 2017 Nov./Dez: 70(6): 1220-1226. Disponivel em: https://pesquisa.bvsalud.org/portal/resource/pt/biblio898321

10. Barbosa ACS, Luiz FS, Friedrich DBC, Püschel VAA, Farah BF, Carbogim FC. Perfil de egressos de Enfermagem: competências e inserção profissional. Revista Latino-Americana de Enfermagem, 2019 Out:27. Disponivel em: https://pesquisa.bvsalud.org/portal/resource/pt/biblio1043072

11. Cambiriba TFC, Ferronato AF, Fontes KB. Percepções de egressos de enfermagem frente a inserção no mercado de trabalho. Arquivos de Ciências da Saúde da UNIPAR, 2014 Jan/Abr: 18 (1): 27-32. Disponivel em: https:// pesquisa.bvsalud.org/portal/resource/pt/lil-761397

12. Colenci R, Berti HW. Formação profissional e inserção no mercado de trabalho: percepções de egressos de graduação em enfermagem. Revista da Escola de Enfermagem da USP, 2012 Fev: 46 (1):158-166. Disponivel em: https://pesquisa.bvsalud.org/portal/resource/pt/mdl-22441280

13. Canever BP, Gomes DC, Jesus BH, Spillere LB, Prado ML, Backes VMS. Processo de formação e inserção no mercado de trabalho: uma visão dos egressos de enfermagem. Revista Gaúcha de Enfermagem, 2014 Mar: 35 (1): 87-93. Disponivel em: https://pesquisa.bvsalud.org/ portal/resource/pt/mdl-24930277 Recuperado em: https://seer.ufrgs.br/ RevistaGauchadeEnfermagem/article/view/43279

14. Silva ANC, Moreira DP, Freitas CMA, Teixeira AKS, Pinheiro ARM. Estágio extracurricular de Enfermagem: estratégia para a formação profissional. Enferm. foco (Brasília), 2019: 10 (4): 129-135. Disponivel em: https://pesquisa.bvsalud.org/portal/resource/pt/biblio-1052819

15. Costa TV, Guariente MHDM. Enfermeiros egressos do currículo integrado: inserção e atuação profissional. Rev. Enferm UFPE on line, 2017 Jan: 11 (1): 77-85. Disponivel em: https://pesquisa.bvsalud.org/portal/ resource/pt/biblio-1031848

16. Brasil. Ministério da Educação. Resolução $\mathrm{CNE} / \mathrm{CES} \mathrm{n}^{\circ}$. 3, de $7 / 11 / 2001$. Institui Diretrizes curriculares nacionais do curso de graduação em enfermagem. Diário Oficial da união, 09 nov. 2001; Seção 1. 